\title{
PSICOTERAPIA COGNITIVO ANALÍTICA Y TRASTORNOS DE LA PERSONALIDAD: REVISIÓN COGNITIVE ANALITIC PSYCHOTHERAPY AND PERSONALITY DISORDERS: REVIEW
}

\author{
Laura Rodríguez Moya ${ }^{\mathrm{a}}$ Y Cristina Fernández Belinchón ${ }^{\mathrm{a}}$ \\ a Psicóloga Clínica. Centro de Atención Integral a Drogodependientes CAID Sur, de la Comunidad \\ de Madrid.laura_moya@hotmail.com
}

Cómo referenciar este artículo/How to reference this article:

Rodríguez-Moya, L. y Fernández-Belinchón, C. (2013). Psicoterapia cognitivo analítica y trastornos de la personalidad: revisión. [Cognitive analitic psychotherapy and personality disorders: review]. Acción Psicológica, 10(1), 65-74. http://dx.doi.org/10.5944/ap.10.1.7034

\section{Resumen}

En los años 80 y 90, A. Ryle desarrolló un modelo de tratamiento denominado Psicoterapia Cognitivo Analítica (PCA). Con un enfoque integrador, breve y focal, la PCA se ha aplicado a diversas patologías, siendo su uso más frecuente con pacientes con trastorno de personalidad, sobre todo con trastorno límite. Estos pacientes poseen un número de disfunciones metacognitivas que dificultan el proceso de darse cuenta de sus diferentes estados mentales y de sus componentes. A través del modelo de Múltiples Estados del Self, el objetivo es ayudar al paciente a identificar los procesos disfuncionales o restrictivos del self que está experimentando y dotarle de recursos para que pueda sustituirlos por otros más adaptativos.

Palabras clave: Psicoterapia Cognitivo Analítica; Trastorno Límite de Personalidad; Modelo de Múltiples Estados del Self.

\begin{abstract}
The Cognitive Analytic Therapy is a kind of integrative, brief, focus and action psychotherapy,
\end{abstract}

developed by A. Ryle in England in the 80's and 90's. At present, is a treatment reference model in that country and is frequently used to treat personality disorders, especially borderline disorder. These patients have a number of metacognitive dysfunctions which difficult the processes of awareness of the different mental states and their components. Through the model of Multiple Self-States, the goal is to help the patient to identify the self's dysfunctional processes which he is experiencing and provide him resources to substitute them for other more adaptive.

Keywords: Cognitive; Analitic Psychotherapy; Borderline Personality Disorder; Model of Multiple Self-States.

\section{Introducción: estado actual de la investigación}

El interés por los Trastornos de Personalidad (TP) ha ocupado, desde el siglo pasado, un lugar predominante en el ámbito de la salud mental. Los motivos principales de dicho interés han sido: su desarrollo temprano y su carácter evolutivo crónico, la alta disfuncionalidad 
psicosocial que lo acompaña, la frecuente comorbilidad con otros trastornos, y su alta incidencia en la población. Cada vez es mayor el número de pacientes con problemas interpersonales crónicos (trastornos de personalidad) que acuden a consulta en las unidades de salud mental (Echeburúa y De Corral, 1999). Todos los esfuerzos científicos y clínicos deben ir dirigidos a desarrollar tratamientos eficaces que puedan dar solución a los problemas psíquicos de estas personas. Éste es uno de los retos de futuro más importantes de la psicología clínica.

La eficacia de la psicoterapia en pacientes con un diagnóstico de Trastorno de Personalidad (TP) siempre ha sido motivo de controversia. En la actualidad, todavía no se han encontrado datos consistentes que indiquen que un tratamiento psicológico específico pueda considerarse «bien establecido» ni para los trastornos de personalidad en general ni para ninguno de los diversos TP en particular (Quiroga y Errasti, 2001). No obstante, se han desarrollado una gran variedad de modelos psicoterapéuticos desde diferentes enfoques teóricos que intentan arrojar luz en este sentido y que están obteniendo buenos resultados.

Uno de estos modelos psicoterapéuticos ha sido la Psicoterapia Cognitivo Analítica (PCA) de A. Ryle, diseñada por este autor en Inglaterra entre los años 80 y 90 (Ryle et al., 1980, 1990, 1991, 1995a, 1997a, 2000, 2006, 2007). Los rasgos distintivos de este modelo de psicoterapia son su carácter integrador, breve, focal y de acción. En la actualidad es uno de los modelos de referencia para el tratamiento de los TP en el Reino Unido y ha alcanzado un notable desarrollo en el resto de Europa. En España se ha extendido gracias a los trabajos de Mirapeix (1994, 1997, 2006).

\section{La PCA: bases teóricas}

Ryle (1991) tomó como punto de partida el estudio del «self». Empezó a interesarse por el modo en que cada individuo construía el propio self y a los demás (Ryle y Lunghi, 1969).

Inicialmente, se basó en el psicoanálisis, en concreto en la teoría de las relaciones objetales de Fairbain (1952). Según esta teoría, el ser humano tiene una necesidad primaria de objetos (de amor y de odio) que el "Yo» busca para encontrar respuesta a su necesidad de relación.

Posteriormente, comenzó a investigar con la técnica de la rejilla de Kelly (1955), instrumento diseñado por dicho autor para estudiar las relaciones interpersonales. Según Kelly, las personas construyen su mundo personal mediante su interpretación de los acontecimientos. Cada individuo aprehende la realidad de acuerdo con sus sistemas de construcción, que dependen a su vez del tipo de supuestos en los que se basa y los valores que le asigna a las cosas.

Ryle también incorporó conceptos de la teoría de esquemas personales de Horowitz $(1988,1991)$. Según esta teoría, cada individuo tiene un repertorio con múltiples esquemas propios. Cuando se produce un cambio en la realidad del sujeto, éste debe integrar este nuevo evento en la memoria a través de la modificación de ciertos esquemas para que concuerden las expectativas internas con la nueva realidad.

Por otro lado, Ryle se apoyó en modelos cognitivos y conductuales, e incorporó la relación entre cognición-emoción-conducta-resultado, el «auto-monitoreo» (Beck, 1976) y la idea de que muchos problemas pueden explicarse siguiendo este tipo de secuencias (sin aludir a procesos inconscientes). Ryle (1982) denominó posteriormente a este proceso $\mathrm{Mo}$ delo de Procedimiento Secuenciado (MPS).

Más tarde se interesó por la psicología evolutiva y la teoría del apego (Bowlby, 1988), sobre todo por la idea de que los vínculos afectivos tempranos (figuras de apego parentales) influyen en el desarrollo de los modelos operativos internos (esquemas personales y de relación) del sujeto, en el desarrollo de la personalidad y en sus futuras relaciones en la edad adulta.

Así mismo, se vio influenciado por la psicología rusa, en concreto por las ideas de Vygotski (1978), quien destacó el papel de la cultura y el contexto social en los que el niño crece 
como guías en el proceso de aprendizaje y en la formación de los procesos mentales superiores; y por el diálogo interpersonal e interno de Bakhtin (1981), según el cual, nos desarrollamos a través de la interacción y comunicación con los demás y la propia conciencia nace a partir de la conciencia del otro (Bakhtin,1981).

Finalmente, del modelo bio-psico-social (Engel, 1977,1980) y la teoría general de sistemas (Von Bertanlannfy, 1965) adoptó la idea del individuo como un sistema global en el que los diferentes elementos cognitivos, afectivos, relacionales, biológicos, históricos y socioculturales se relacionan entre sí, y pueden estar afectados de forma particular.

\section{La PCA: modelo integrador, breve, focal y de acción}

Tomando como punto de partida todas estas aportaciones teóricas previas, Ryle describió la PCA (1991) como un modelo de terapia «integrador», que incorpora elementos de diferentes enfoques teóricos y los combina dentro de un modelo de intervención con una estructura y protocolo determinados.

El formato inicial de la PCA fue «breve», de 16 sesiones, para que pudiera ser aplicada en el sistema público de salud y permitiera atender a un gran número de pacientes. Posteriormente, este modelo se ha ido adaptando a diferentes contextos y patologías. Existen formatos de mayor duración, con sesiones adicionales, dirigidos al tratamiento de patologías más graves (por ejemplo, el trastorno límite de personalidad), y también formatos más breves, con menor número de sesiones, para pacientes con una patología más leve. Así mismo, aunque el formato inicial iba dirigido al tratamiento individual, en la actualidad se ha extendido su utilización a terapias de familia, pareja y de grupo, y se ha implementado en diferentes contextos clínicos (ambulatorio, hospital de día, etc.).

La Psicoterapia Cognitivo Analítica (PCA) es una terapia "analítica», porque se basa en la idea de que existen aspectos que no son accesi- bles al sujeto de forma consciente y que a través de la terapia pueden ser accesibles a este nivel. Es también una terapia "cognitiva», porque se centra en la capacidad del sujeto para pensar y reflexionar sobre sí mismo y adquirir mayor control sobre sus propias cogniciones, emociones y conductas.

La PCA es una psicoterapia «focal» $\mathrm{y}$ «de acción». El terapeuta toma un papel activo y focaliza el trabajo terapéutico en ayudar al paciente a ser consciente de sus patrones de interacción disfuncionales (cognición-afecto-conducta-consecuencia) y a encontrar los procedimientos adecuados para cambiar estos patrones y sustituirlos por otros más adaptativos. (Ryle y Kerr, 2006).

\section{Conceptos básicos de la PCA}

\section{Concepto de self}

La PCA entiende que el self es "social» (Ryle y Kerr, 2006), y que es el resultado de la interacción temprana entre el niño y sus cuidadores, en un contexto y cultura determinados.

El niño internaliza estas interacciones con los demás y su entorno en forma de representaciones mentales denominadas «estados mentales»y "patrones de rol recíprocos». De esta forma aprende a adoptar, actuar, pensar, o sentir de forma diferente en función de las personas o circunstanciales contextuales y temporales en las que se encuentre. Estos estados mentales y patrones de rol coexisten dentro de un «self coherente e integrado». De manera que, aunque actué de formas diferentes, el individuo se siente un ser único.

\section{Falso self}

Aquel individuo cuyo sentido del self es vivido como una falta de autenticidad o como «no estar en diálogo» con el otro (Ryle y Kerr, 2006). Su sentido del self depende excesivamente de la respuesta de los demás y apenas tiene conciencia de sus propios sentimientos. 


\section{Desarrollo anormal del self}

«El desarrollo anormal del self se produce a través de la internalización de procedimientos de rol disfuncionales, el desarrollo de procedimientos de rol evitantes, defensivos y sintomáticos o las rupturas en la integración de los procesos del self»(Ryle y Kerr, 2006).Es decir, cuando el sujeto internaliza formas de interactuar o estar con los otros que resultan desadaptativas o cuando no existe una coherencia o continuidad en esos patrones de comportamiento.

El desarrollo del self puede verse afectado a través «de la internalización de procedimientos de rol recíprocos negativos o maladaptativos» o a través de la "sustitución de éstos por procedimientos sintomáticos o restrictivos» (Ryle y Kerr, 2006). Estos dos tipos de experiencias interpersonales tempranas (por ejemplo: padres excesivamente críticos o culpabilizadores) contribuyen a la construcción de un autoconcepto desadaptativo, lo que lleva a estos sujetos a reproducir en la vida adulta roles negativos aprendidos en la infancia (ej: sumisión, victimización o agresión). El desarrollo del self puede verse afectado también a través de «la disociación, inducida por la ansiedad o por el trauma» (Ryle y Kerr, 2006). Lo que quiere decir que, la dificultad para integrar recuerdos o sentimientos relacionados con un trauma infantil, puede impedir el desarrollo de un self coherente y unitario, generando diferentes self que funcionan de forma total o parcialmente disociada.

Estos tres tipos de experiencias tempranas disfuncionales pueden encontrarse en cualquier trastorno de personalidad, pero sobre todo aparecen en el Trastorno Límite de Personalidad (TLP), especialmente el tercero (Ryle, 1993, 1995c, 1997a y 1997b, 2000). Según Ryle (2006), gran parte de la fenomenología del TLP es fruto de la existencia de un gran número de estados del self parcialmente disociados y extremos, de modo que los metaprocedimientos que normalmente ayudan a integrar los diferentes estados del self están alterados o no se han desarrollado debidamente; en ocasiones también se puede dar cambios muy bruscos y confusos entre los estados, lo que provoca una gran inestabilidad.

\section{Modelo de los múltiples estados del self y TLP: Estados mentales y Procedimientos de roles recíprocos}

El concepto de «estado mental» hace referencia a la auto-representación o percepción subjetiva de uno mismo en cada momento. Es un concepto que puede asimilarse al de «Modelo Operativo Interno» de Bowlby (1988). El estado mental de un individuo estaría formado por cogniciones, emociones y conductas.

Los «roles recíprocos» son los patrones de relación interpersonal o repertorios de conducta que el sujeto incorpora en el proceso de aprendizaje interpersonal. Son las formas que tiene cada uno de estar con los demás (Ryle, 1985). Se adquieren bien de forma directa o de forma vicaria. Desde el nacimiento, el niño va construyendo significados en función de los roles que asumen él y los demás en cada situación. De esta manera los niños aprenden a manejar múltiples relaciones de rol mientras que interactúan con otros (Ryle y Kerr, 2006).

Ryle opina que en los trastornos de personalidad existe una disfunción de los estados mentales y de los patrones de rol recíprocos. En concreto, en el Trastorno Límite de Personalidad (TLP), existen una serie de disfunciones metacognitivas que dificultan los procesos de toma de conciencia de los diferentes estados mentales y de sus componentes.

Estos pacientes reproducen en la vida adulta patrones tempranos extremos, normalmente derivados de relaciones de abuso o negligencia con cuidadores tempranos. Como consecuencia de dichos cuidados disfuncionales o de una disociación parcial inducida por el trauma, las habilidades metacognitivas que permiten identificar estados mentales y pasar de uno a otro de forma adaptativa no se desarrollan correctamente. El resultado es que co-existen roles recíprocos diferentes, desconectados, y parcial o totalmente disociados. Como consecuencia, la capacidad de autorreflexión se encuentra muy dañada, lo que disminuye su capacidad 
para responsabilizarse de sus conductas o aprender de la experiencia. (Ryle y Kerr, 2006).

A partir de estudios realizados con la técnica de la rejilla en pacientes con un trastorno límite de la personalidad, Ryle determinó 11 estados mentales en estos pacientes, de los cuales 10 son considerados dañinos (Ryle y Kerr, 2006). El grupo de Mirapeix (2007) hace la siguiente adaptación:

Estado ok. Estoy más o menos normal en mi vida cotidiana.

Estado de víctima. Siento que los demás abusan de mí, no me respetan ni me cuidan, me amenazan o me maltratan.

Estado de "cargo con todo". Hago lo que tengo que hacer, cumplo con las expectativas de los demás sin disfrutar de ello.

Estado de rabia. Me siento fuera de control, peligroso hacia mí y/o hacia otros.

Estado vengativo. Me siento enfurecido, violento, envidioso, deseando vengarme.

Estado de "anestesiado». Me siento insensible ante las emociones, indiferente hacia los demás, desapegado.

Estado abusadorlexplotador. Me siento insensible, sin compasión, despectivo hacia los demás, deseando hacerles daño o hacerme daño a mí mismo.

Estado acelerado. Me siento hiperactivo, con energía, a tope.

Estado de "nirvana». Me siento feliz, protegido, seguro, cercano a los demás.

Estado de superioridad. Siento que soy mejor que los demás, especial, digno de admiración.

Estado protector. Me siento capaz de ayudar a otros, necesario, con capacidad de cuidar los demás.

\section{Patrones disfuncionales de pensamiento y conducta}

Dentro de su modelo de Múltiples Estados del Self, Ryle (2006) también describe tres ti- pos de patrones disfuncionales de pensamiento $\mathrm{y}$ conducta (semejante a las distorsiones cognitivas de Beck) que aparecen con frecuencia en los trastornos de personalidad y en otros cuadros psicopatológicos:

1) Dilemas: patrones de pensamiento rígidos, que impiden la modificación de las creencias del sujeto porque expresan las posibilidades de acción en forma de elecciones polarizadas, de manera que las únicas opciones aparentemente posibles son seguir la alternativa menos desagradable $\mathrm{u}$ oscilar entre ellas (por ejemplo: o me relaciono con los demás, y me expongo a que me puedan hacer daño, o me mantengo distante y a la defensiva pero me siento sólo).

2) Trampas: Patrones de pensamiento rígidos que mantienen las creencias negativas de uno mismo, mediante la generación de formas de conducta que conducen a consecuencias (respuesta de los demás) que confirman las creencias iniciales (profecía autocumplida).

3) Pegas: Patrones de pensamiento según los cuales el sujeto abandona o sabotea sus propios objetivos porque cree que su logro podría ser peligroso para sí mismo o para los demás (encontramos el ejemplo en el saboteo de las cosas buenas como si uno no las mereciera).

\section{Modelo de tratamiento}

Tal y como hemos comentando anteriormente, la Psicoterapia Cognitivo Analítica (PCA) es una terapia "analítica», porque parte de la idea de que existen aspectos que no son accesibles al sujeto en un plano consciente y que a través de la terapia pueden ser accesibles a nivel consciente. Pero a la vez, es también una terapia "cognitiva», porque se centra en la capacidad del sujeto para pensar y reflexionar sobre sí mismo y adquirir un mayor control sobre sus propias acciones. La PCA es también una psicoterapia «focal» $\mathrm{y}$ «de acción». El terapeuta toma un papel activo y actúa como un «andamiaje» para el paciente en su nuevo proceso de aprendizaje. El «foco terapéutico» con- 
siste en ayudar al paciente a que sea consciente e identifique sus patrones de interacción disfuncionales (cognición-afecto-conductaconsecuencia) y a que pueda cambiar estos patrones y sustituirlos por otros más adaptativos. (Ryle y Kerr, 2006). La meta final no es modificar las creencias del paciente sobre la experiencia inmediata, sino ayudarle a comprender su propio modo de elaborarlas para que así pueda reformularlas él mismo. (Ryle, 1982; Ryle y Kerr, 2006).

\section{Fases del tratamiento en PCA}

La psicoterapia cognitivo analítica fue diseñada originalmente para ser «breve» (16 sesiones). Pero aplicada a los Trastornos de Personalidad, su duración se ha extendido. Independientemente del número de sesiones que se utilicen en cada caso, las fases del proceso siempre son las siguientes:

\section{1. ${ }^{a}$ Fase de indicación y Psicopedagogía}

En esta fase se analiza cuál es el problema y la motivación del paciente para cambiar y acudir a terapia. También se valora si es un paciente susceptible de beneficiarse de la PCA. En esta fase es especialmente importante dedicar tiempo al establecimiento de alianza terapéutica.

\section{2. ${ }^{a}$ Fase de evaluación: aplicación de cuestionarios específicos}

\section{Cuestionario de Psicoterapia}

Se da a los pacientes al final de la primera sesión para que lo completen en casa. Recoge explicaciones y ejemplos de dilemas, trampas y pegas. El paciente tiene que reflexionar sobre cuáles de ellos coinciden consigo mismo. Al inicio de la siguiente sesión se trabajan con el terapeuta y también a lo largo de toda la terapia. El cuestionario también recoge instrucciones para la auto-monitorización de estados anímicos y de síntomas. Con esta información el terapeuta comienza a elaborar un listado de conductas o procedimientos problemáticos del paciente.

Cuestionario de Estructura de

Personalidad (Personality Structured

Questionnaire PSQ) (Pollock, Broadbent,

Clarke, Dorian, y Ryle, 2001)

Este instrumento consta de 8 ítems que evalúan la capacidad de integración de la personalidad.

Procedimiento de descripción de estados (State Description Procedure, SDP) (Bennett, Pollock y Ryle, 2005)

Este instrumento se divide en 2 partes. La 1. ${ }^{a}$, denominada: Identificando sus estados mentales, describe los 11 estados mentales básicos (Ryle y Kerr, 2006). En ella, el sujeto debe señalar aquellos estados con los que se identifica. Previamente se le explica lo que significa «estado mental» desde el modelo de la PCA. Así mismo, el sujeto puede añadir otros estados mentales que experimente al margen de esos 11, al final de la lista. En la 2. ${ }^{a}$ parte: Describiendo mis estados mentales, el sujeto debe describir los estados mentales que ha señalado con mayor detalle. Para cada estado debe especificar: 1) cómo se siente en su vida desde ese estado (satisfecho, seguro, despreciable etc.); 2 ) cómo es su actitud hacia los demás desde ese estado (respetuoso, exigente, temeroso, violento, etc.); 3) cómo es la actitud de los demás hacía él cuando se encuentra en ese estado mental (respetuosa,, exigente, cuidadora, controladora, despectiva, etc.); 4) otras características de cada estado mental (con qué frecuencia ha experimentado ese estado mental en los últimos 6 meses, cuánto tiempo dura normalmente, cómo empieza normalmente ese estado, cómo finaliza normalmente ese estado, qué le hace entrar en ese estado, qué le hace salir de ese estado, si cuando se encuentra en ese estado recuerda otros de los estados que ha descrito, etc.) (Mirapeix, 2007). 
Con este cuestionario el terapeuta obtiene información sobre los estados mentales del paciente. Pero además, la cumplimentación de este cuestionario ayuda al paciente a que reflexione sobre sus estados mentales y las consecuencias que derivan de cada uno. Para reforzar esta capacidad de autoobservación y autorreflexión sobre los propios estados del self, se suelen utilizar autorregistros durante todo el periodo intersesiones, Esta estrategia ayuda al paciente a ganar control sobre los cambios bruscos y dañinos y poder integrar los self disociados.

A través de esta metodología de evaluación, el terapeuta construye con el paciente narrativas sobre lo que suele ocurrir en las diferentes situaciones a las que el paciente se enfrenta en interacción con otros.

\section{3. ${ }^{a}$ Reformulación}

\section{Carta de reformulación}

Para mostrarle al paciente cómo se han formado los problemas, el terapeuta le devuelve una reconstrucción de su historia, en forma de «carta» (Ryle y Kerr, 2006).

El formato de esta carta es el siguiente: 1) En primer lugar, el terapeuta le devuelve un resumen breve del relato que el paciente le ha contado (motivo de consulta e historia vital) en las sesiones de evaluación; 2) En segundo lugar, le reformula la relación que existe entre sus experiencias del pasado y sus procedimientos actuales. El terapeuta le señala las trampas, dilemas, y pegas o procedimientos de rol recíproco desadaptados que ha detectado y centra los objetivos de la terapia en la modificación de los mismos.

\section{Reformulación diagramática}

Para la reformulación de los procedimientos problemáticos detectados, la PCA utiliza además un formato gráfico: los diagramas de flujo (Ryle y Kerr, 2006).Representa las trampas, los dilemas, las pegas y los patrones de rol recíproco disfuncionales actuales, en forma de diagramas de flujo. La representación, visual y en forma de esquema, favorece la comprensión del paciente sobre lo que le está sucediendo, ya que le permite ver las relaciones que se establecen entre cada uno de estos elementos y las consecuencias que se derivan de ellos. El terapeuta le muestra al paciente cómo los diagramas se integran en un modelo denominado de «Procesamiento Secuenciado Global» (Ryle y Kerr, 2006). Este modelo, refleja el carácter repetitivo y disfuncional de los procedimientos que el paciente utiliza en diferentes contextos y situaciones de su vida, y cómo eventos del pasado han influido en comportamientos actuales. Con el Modelo de Procesamientos Secuenciado Global, el terapeuta ayuda al paciente a reflexionar sobre cuáles de estos estados mentales o roles son disfuncionales (causantes de psicopatología), pero también sobre cuáles no lo son (es decir, estados mentales o roles que son adaptativos y que por lo tanto se favorecerá que mantenga).

En el caso de los trastornos de personalidad, este instrumento es especialmente útil, sobre todo en el caso del TLP. Los diagramas de flujo y el Modelo de Procesamiento Secuenciado, sirven como guía a estos pacientes para aprender a diferenciar entre «estados mentales del yo» y «estados mentales de los otros», pudiendo recapacitar sobre el impacto que sus comportamientos tienen en los otros, y entendiendo la relación entre aspectos del pasado (infancia, vínculos de apego con los cuidadores u otros significativos) y el presente. En el caso de los pacientes con TLP, el reconocimiento de los estados mentales y de los cambios de uno a otro será un objetivo prioritario.

Una vez definidos los diagramas integrados en el Modelo de Procesamiento Secuenciado, se procede a realizar con el paciente "reformulaciones narrativas» de todos estos aspectos. A través de los mismos diagramas, el terapeuta ayuda al paciente a reflexionar y desarrollar estados mentales alternativos que, en función del contexto y según sus intenciones u objetivos, puedan resultar más eficaces o beneficiosos para sí mismo. En las sesiones también se suelen utilizar otras técnicas: intervenciones transferenciales, role-playing, técnicas conductuales 
como la exposición a situaciones temidas o experimentos conductuales en los que el paciente debe poner en marcha procedimientos alternativos a los disfuncionales, ensayar formas de manejo de estados emocionales negativos, ensayar pensamientos alternativos y formas de diálogo consigo mismo, ejercicios de relajación física...; también se incluyen tareas para casa que los pacientes deben realizar en el periodo intersesiones para mejorar su autoconciencia. Todas estas técnicas se incluyen en el diagrama de flujo y se ponen en relación con los estados emocionales y los patrones de rol recíproco. De manera que el paciente pueda integrar dichas alternativas para poner en marcha cada vez que identifique un patrón de pensamiento-conducta o emoción disfuncional.

La meta final no es modificar las creencias del paciente sobre la experiencia inmediata, sino ayudarle a comprender su propio modo de elaborarlas para que así pueda reformularlas él mismo de forma cada vez más autónoma (Ryle, 1982; Ryle y Kerr, 2006). Este tipo de pacientes, no han podido internalizar el rol del autocuidado (Ryle y Kerr, 2006). La terapia se convierte en un medio a través del cual aprenden a cuidarse a sí mismos, separando pasado y presente, de forma que puedan sentirse adultos libres y responsables.

\section{4. ${ }^{a}$ Carta de despedida}

En la penúltima o última sesión, terapeuta y paciente se intercambian cartas de despedida. La carta del terapeuta, recoge todos aquellos aspectos trabajados en la terapia. Describe los objetivos alcanzados así como los que aún requieren de trabajo adicional por parte del paciente. El objetivo de la carta, al igual que el de toda la terapia, es ayudar al paciente a internalizar la experiencia para que pueda continuar con el proceso él solo. En la carta del paciente, se le ofrece un espacio para que él reflexione sobre todo lo sucedido en el proceso de terapia (Ryle y Kerr, 2006). Suele establecerse una sesión de seguimiento a los tres meses.

\section{Evidencias empíricas de la PCA y el tratamiento del TLP}

Como decíamos al inicio de este artículo, en la actualidad existe una gran controversia en torno a la efectividad de la psicoterapia en los trastornos de personalidad. Si bien, la PCA todavía no ha sido incluida como «tratamiento eficaz» en este ámbito, se han publicado diversos estudios sobre la evidencia empírica de este enfoque (Dunn, 1994; Marloe y Ryle, 1995; Pollock, 1996; Ryle, 1997a, 1997b). En España destacan los trabajos de Mirapeix (1994, 1997, 2006). Aquellos interesados en profundizar en este enfoque pueden visitar la página web de la Asociación para la Terapia Cognitivo Analítica (ACAT) en el Reino Unido: http://www.acat.org. uk, o consultar la página en castellano en la página del Instituto de Estudios Psicoterapéuticos http://www.iepsi.org.

\section{Referencias}

Bakhtin, M. M. (1981). The Dialogic Imagination: Four essays. Austin: U. Texas Press.

Bateman, A. W., Ryle, A., Fonagy, P. y Kerr, I. B. (2007). Psychotherapy for Borderline Personality Disorder: Mentalization based therapy and Cognitive Analytic Therapy compared. International Review of Psychiatry, 19(1), 51-62.

Beck, A. T. (1976). Cognitive Therapy and Emotional Disorders. Nueva York: International Universities Press.

Bennet, D., Pollock, P. y Ryle, A. (2005). The States Description Procedure: the use of guided selfreflection in the case formulation of patients with Borderline Personality Disorder. Clinical Psychology \& Psychotherapy, 12(1), 50-57.

Bowlby J. (1988). A secure base: clinical applications of attachment theory. Londres, UK: Routledge.

Dunn, M. (1994). Variations in cognitive analytic therapy in the treatment of a severely disturbed patient. International Journal of Short-Term Psychotherapy, 9, 83-92.

Echeburúa, E. y De Corral, P. (1999). Avances en el tratamiento cognitivo-conductual de los Trastornos de Personalidad. [Advances in cognitive 
behavioral treatment of personality disorders]. Análisis y Modificación de Conducta, 25(102), 585-614.

Engel, G. L. (1977). The need for a new medical model a challenge for biomedicine. Science, 136, 126-129.

Engel, G. L. (1980). The clinical application of the biopsychosocial model. American Journal of Psychiatry, 137, 535-544.

Fairbain,W. R. (1952). Estudio psicoanalítico de la personalidad. [Psychoanalitic study of personality]. Buenos Aires, Argentina: Hormé

Horowitz, M. J. (1979). States of Mind. New York: Plenum.

Horowitz, M. J. (1987). States of mind: Configurational analysis of individual psychology (2. ${ }^{\mathrm{a}} \mathrm{Ed}$.). New York: Plenum.

Horowitz, M. J. (1988). Psychodinamics and cognition. Chicago: The University of Chicago Press.

Horowitz, M. J. (1991). Person schemas and maladaptative interpersonal patterns. Chicago: The University of Chicago Press.

Kelly, G. A. (1955). The Psychology of Personal Constructs. Nueva York: Norton.

Marlowe, M. y Ryle, A. (1995). Cognitive Analytic Therapy of borderline personality disorder: Theory and practice and the clinical and research uses of the self states sequential diagram. International Journal of Short-Term Psychotherapy, 10, 21-34.

Mirapeix, C. (1994). Psicoterapia Cognitivo Analítica: un paradigma de integración en psicoterapia. [Cognitive Analytic Psychotherapy: a paradigm of integration in psychotherapy]. Revista de psicoterapia, 20, 5-44.

Mirapeix C. (1997). De la multicausalidad biopsico-social a una concepción integradora de la psicoterapia [From multi-causal biopsychosocial model to an integrated conception of psychotherapy]. Archivos de Neurobiología, 60(1), 1-3.

Mirapeix, C, Uríszar-Aldaca, M., Landín, S. y Nieto, A. (2006). Tratamiento multicomponente de orientación cognitivo analítica del trastorno límite de personalidad. [Multicomponent cognitive analytic treatment of borderline personality disorder]. Psiquiatría.com, 10(1).
Mirapeix, C. (2007). Seminario Tratamiento actual de los Trastornos de Personalidad [Current treatment of personality disorders]. Agencia Laín Entralgo, Madrid, 31 enero-2 febrero.

Pollock, P. H. (1996). Clinical issues in the cognitive analytic therapy of sexually abused women who commit violent offences against their partners. British Journal of medical Psychology, 69, 117-127.

Pollock, P. H., Broadbent, M., Clarke, S., Dorian, A. y Ryle, A. (2001). The Personality Structured Questionnaire (PSQ): A measure of the multiple self-states model of identity confusion in cognitive analytic therapy. Clinical Psychology and Psychotherapy, 8, 59-72.

Quiroga, E. y Errasti, M. (2001). Tratamientos psicológicos eficaces para los Trastornos de Personalidad. [Effective psychological treatments for Personality Disorders]. Psichotema, 13(3), 303-405.

Ryle, A. y Lunghi. M. E. (1969). The measurement of relevant change after psychotherapy: use of repertory grid testing. British Journal of Psychiatry, 115(528), 1297-304.

Ryle, A. (1980). Some measures of goal attainment in focused, integrated, active psychotherapy: a study of fifteen cases. British Journal of Psychiatry, 137, 475-486.

Ryle, A. (1982). Psychotherapy: A Cognitive Integration of Theory and Practice. Londres, UK: Academy Press.

Ryle, A. (1985). Cognitive theory, object relations and the self. British Journal of medical Psycho$\log y, 58,1-7$.

Ryle, A. (1990). Cognitive Analytic Therapy: Active Participation in Change. Chichester: Wiley

Ryle, A. (1991). Object relations theory and activity theory; a proposed link by way of the procedural sequence model. British Journal of medical Psychology, 64, 307-316.

Ryle, A. (1993). The integrative effect of reformulation: cognitive analytic therapy with a patient with borderline personality disorder. British Journal of medical Psychology, 66, 249-258.

Ryle, A. (1995a). Cognitive Analytic Therapy, Developments in Theory and Practice. Chichester: Wiley

Ryle, A. (1995b). Holmes on Bowlby and the future of pshychotherapy: a response. British Journal of Psychotherapy, 11, 448-452. 
Ryle, A. (1995c). Cognitive Analytic Therapy of borderline personality disorder: Theory and practice and the clinical and research uses of the self states sequential diagram. International Journal of short-term Psychotherapy, 10, 21-34.

Ryle, A. (1997a). Cognitive Analytic Therapy and Borderline Personality Disorder: The Model and the Method. Chichester: Wiley.

Ryle, A. (1997b). The structure and development of borderline personality disorder; a proposed model. British Journal of Psychiatry, 170, 82-87.

Ryle, A. y Golynkina, K. (2000). Effectiveness of time-limited cognitive analytic therapy of borderline personality disorder: factors associated with outcome. British Journal of Medical Psychology, 73(2), 197-210.
Ryle, A. (2004). The contribution of cognitive analythic therapy to the treatment of borderline personality disorder. Journal of Personality Disorders, 18(1), 3-35.

Ryle, A. y Kerr, I. B. (2006). Psicoterapia Cognitivo Analítica: Teoría y práctica. [Cognitive Analytic Psychotherapy. Theory and practice]. Bilbao, España: Descleé de Brower.

Ryle, A. (2007). Investigating the phenomenology of borderline personality disorder with the States Description Procedure: clinical implications. Clinical Psychology and Psychotherapy, 14(5), 329-341.

Von Bertalanffy, L. (1968). General Systems Theory. New York: Brazilie.

Vygotsky, L. S. (1978). Mind in Society: The Development of higher Psychological Processes. Cambridge, MA: Harvard University Press. 